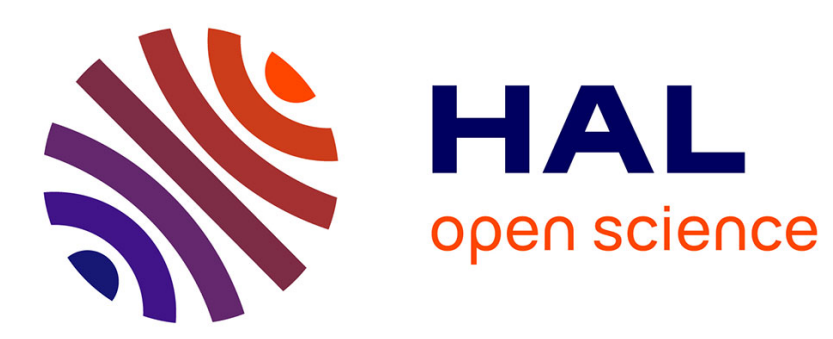

\title{
Etude du réisolement d'un disjoncteur à vide pour impulsion de courant intense haute fréquence
}

\author{
A. Delmas, A. Dongfack, C. Rioux
}

\section{To cite this version:}

A. Delmas, A. Dongfack, C. Rioux. Etude du réisolement d'un disjoncteur à vide pour impulsion de courant intense haute fréquence. Revue de Physique Appliquée, 1987, 22 (8), pp.761-765. 10.1051/rphysap:01987002208076100 . jpa-00245606

\section{HAL Id: jpa-00245606 https://hal.science/jpa-00245606}

Submitted on 1 Jan 1987

HAL is a multi-disciplinary open access archive for the deposit and dissemination of scientific research documents, whether they are published or not. The documents may come from teaching and research institutions in France or abroad, or from public or private research centers.
L'archive ouverte pluridisciplinaire HAL, est destinée au dépôt et à la diffusion de documents scientifiques de niveau recherche, publiés ou non, émanant des établissements d'enseignement et de recherche français ou étrangers, des laboratoires publics ou privés. 


\title{
Etude du réisolement d'un disjoncteur à vide pour impulsion de courant intense haute fréquence
}

\author{
A. Delmas, A. Dongfack et C. Rioux \\ Laboratoire d'Electrotechnique (*) des Universités Paris VI et XI, 91405 Orsay, France
}

(Reçu le 9 février 1987, révisé le 21 avril 1987, accepté le 28 avril 1987)

\begin{abstract}
Résumé. - L'étude est relative aux capacités de récupération de la rigidité diélectrique d'un disjoncteur à vide, après un passage durant $30 \mu$ s d'une impulsion de courant intense pouvant atteindre $20 \mathrm{kA}$. Les temps de réisolement imposés sont de l'ordre de $200 \mu \mathrm{s}$. Des électrodes en molybdène et en cuivre ont été utilisées pour différentes distances de séparation.
\end{abstract}

\begin{abstract}
The article is relative to the vacuum switch recovery capacities, after a crossing during $30 \mu$ s of a high current pulse which can reach $20 \mathrm{kA}$. The fixed recovery times are in order of $200 \mu \mathrm{s}$. Molybdenum and copper electrodes were used for different gaps.
\end{abstract}

\section{Introduction.}

De nombreux travaux portant sur le comportement d'un arc sous vide - et en particulier sur ses capacités de réisolement après extinction - ont déjà donné lieu à de multiples publications $[1,2]$. Ces travaux ont trait généralement à la disjonction de courant pouvant atteindre $10^{5}$ ampères, après un temps de passage (ou bien une période d'oscillations) de l'ordre de quelques ms à quelque $10^{1} \mathrm{~ms}$; d'autre part les variations $\frac{\mathrm{d} I}{\mathrm{~d} t}$ du courant excédent rarement $10^{2} \mathrm{~A} / \mu \mathrm{s}$. On montre expérimentalement que les meilleurs résultats sont obtenus lorsque l'arc interélectrodes est " diffus », c'est-à-dire couvre la quasitotalité de la surface des électrodes sans mettre en œuvre de tache anodique [3]. Dans un tel problème il apparaît que la nature des électrodes joue un rôle non seulement sur l'apparition de taches anodiques $[4,5]$ mais également sur le pouvoir de réisolement de l'espace inter-électrodes après coupure du courant $[6,7]$. Le rapport du courant d'arc maximum à la surface de l'électrode atteint ici quelques ampères $/ \mathrm{mm}^{2}$ voire beaucoup moins dans le cas d'isolement rapide à de très hautes tensions [8]. Les vitesses de réisolement sont alors de quelques kilovolts par microseconde $[8,9]$.

(*) Laboratoire associé au CNRS n 845 .
L'étude exploratrice présentée ici se place dans une optique sensiblement différente. Pour une application ultérieure de transfert d'énergie entre deux inductances avec un rendement élevé $[10,11]$ on a besoin de moyens de disjonctions répétitifs pour lesquels le temps de passage du courant est bref (quelque $10^{-5} \mathrm{~s}$ ), alors que le temps de réisolement peut être relativement long (de l'ordre de $10^{-4} \mathrm{~s}$ ). De plus pour obtenir des capacités énergétiques suffisantes, il faut que les intensités mises en jeu soient importantes, par exemple quelque $10^{4} \mathrm{~A}$. Les vitesses de variation du courant $\frac{\mathrm{d} I}{\mathrm{~d} t}$ sont alors nettement plus élevées que celles rencontrées couramment. Notons également que nous avons ici, contrairement à ce que l'on rencontre dans la quasitotalité des applications, une tension de rétablissement de même polarité que l'impulsion de courant. Compte tenu de ces contraintes, il devenait nécessaire d'effectuer un certain nombre d'expérimentations dont les premières sont rapportées dans le présent article.

Notons que dans le cas d'une disjonction si rapide il ne saurait être question de déplacement mécanique des électrodes: aussi le disjoncteur est de type « éclateur». Dans le cas qui nous concerne, on fait varier divers paramètres géométriques, tels que la distance inter-électrodes, ainsi que la nature des électrodes (cuivre et molybdène). 


\section{Montage expérimental.}

2.1 Disjoncteur. - Il est représenté sur la figure 1. C'est un système de révolution constitué de 3 électrodes :

- 2 électrodes principales (7) et (3) (4).

- 1 électrode de déclenchement en molybdène

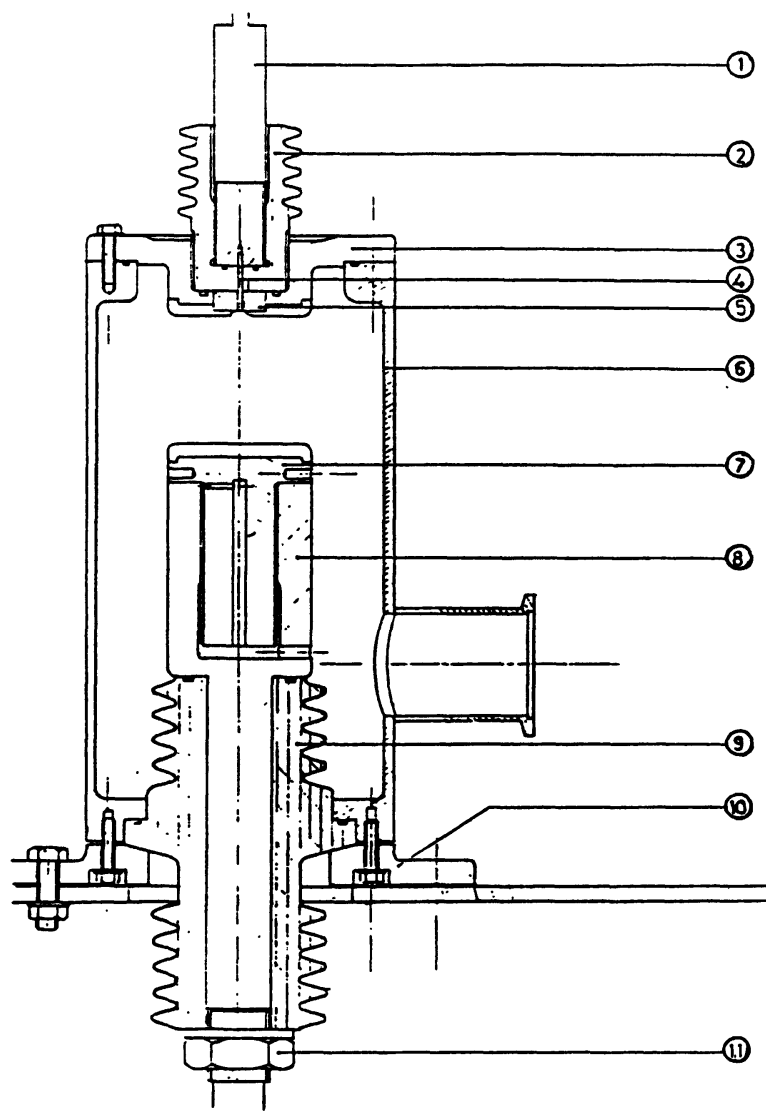

Fig. 1. - Disjoncteur : (1), (4) Déclencheur. (2), (5), (9) Isolant. (6) Enceinte en acier inoxydable. (3) Cathode et support. (7), (8) Anode et support.

[Switch : (1), (4) Trigger system. (2), (5), (9) Insulator. (6) Stainless steel chamber. (3) Cathode and support. (7), (8) Anode and support.]

Les électrodes principales sont planes avec leurs extrémités arrondies (rayon $5 \mathrm{~mm}$ ).

L'électrode de déclenchement, de petit diamètre ( $2 \mathrm{~mm}$ ), est disposée sur l'axe de la cathode, laquelle constitue le potentiel de référence du système de déclenchement. Cette électrode est centrée à l'aide d'une rondelle d'alumine (5). Ce procédé de centrage présente de plus l'avantage de réduire la tension de claquage de l'impulsion d'amorçage avec la cathode. Enfin un jeu de cales intermédiaires permet de régler la distance inter-électrodes.

L'impulsion d'amorçage a une durée d'environ $2 \mu$ s et une tension initiale de 8 à $9 \mathrm{kV}$ pour un courant crête de $10^{3} \mathrm{~A}$ environ.
Le vide est maintenu à une pression inférieure à $10^{-6}$ torr par une pompe à diffusion d'huile.

2.2 Montage ÉlectriQue. - Les conditions de fonctionnement testées étant relativement précises pour l'application considérée on a choisi un montage d'essai particulièrement simple «en puissance », c'est-à-dire sans mettre en jeu les montages dits «pour essais synthétiques » qui sont habituellement utilisés.

L'idée de base consiste à relier l'éclateur à tester à deux capacités égales montées en parallèles, chacune d'elles étant placée en série avec une inductance. Comme les deux inductances sont très différentes, le phénomène complet s'effectue en deux temps :

- dans le premier, le circuit doté de la plus petite inductance sera le siège d'une décharge oscillante rapide du condensateur au travers du disjoncteur. Il sera le générateur de l'impulsion du courant intense qui alimentera l'arc (Fig. 3b). Le deuxième circuit doté d'une inductance beaucoup plus importante, donc d'une période d'oscillation plus grande, verra sa décharge peu développée lors de l'annulation du courant dans le disjoncteur;

- dans le deuxième, le disjoncteur ayant perdu ses propriétés conductrices, le circuit «longue période " va poursuivre sa décharge au travers du premier circuit. La tension aux bornes du disjoncteur, après avoir atteint brusquement une valeur négative à l'extinction, va progressivement croître jusqu'à atteindre une tension crête voisine de la tension de charge des condensateurs. Nous obtenons là le test d'isolement post-arc du disjoncteur. En pratique, l'alimentation en courant du disjoncteur est symétrique. Elle est constituée de deux circuits oscillants identiques (Fig. 2) $C_{0}, L_{0}$, dotées d'une résistance d'amortissement $R_{0}$ permettant d'atténuer l'intensité du pic de tension à l'extinction du courant au niveau du disjoncteur. La période d'oscillation de ce circuit est de $54 \mu \mathrm{s}$. Le circuit permettant le test en tension (comprenant la self $L_{1}$ et les deux capacités $C_{0}$ ) a une période d'oscillation de $620 \mu \mathrm{s}$ via le disjoncteur et de $310 \mu$ s via l'autre circuit oscillant. A l'extinction, la tension remonte de

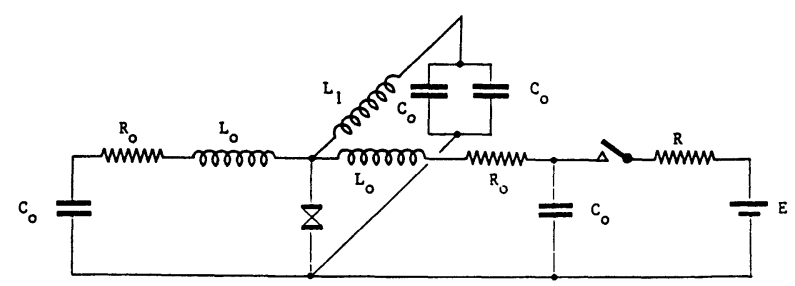

Fig. 2. - Schéma électrique : $R_{0}=1,02 \Omega ; C_{0}=8 \mu \mathrm{F}$; $L_{0}=5,6 \mu \mathrm{H} ; L_{1}=610 \mu \mathrm{H}$.

[Electrical diagram : $R_{0}=1.02 \Omega ; C_{0}=8 \mu \mathrm{F} ; L_{0}=$ $5.2 \mu \mathrm{H} ; L_{1}=610 \mu \mathrm{H}$.] 
- $10 \%$ à près de $100 \%$ de la tension initiale de charge des condensateurs en un temps de $200 \mu \mathrm{s}$ (Fig. 3a). La durée du pic négatif de tension est de $25 \mu \mathrm{s}$.

$5,7 \mathrm{kV}$

a)

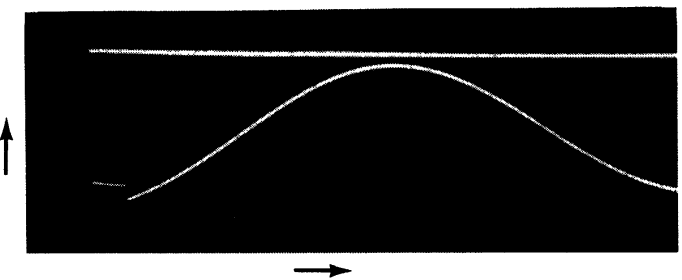

$50 \mu \mathrm{s}$

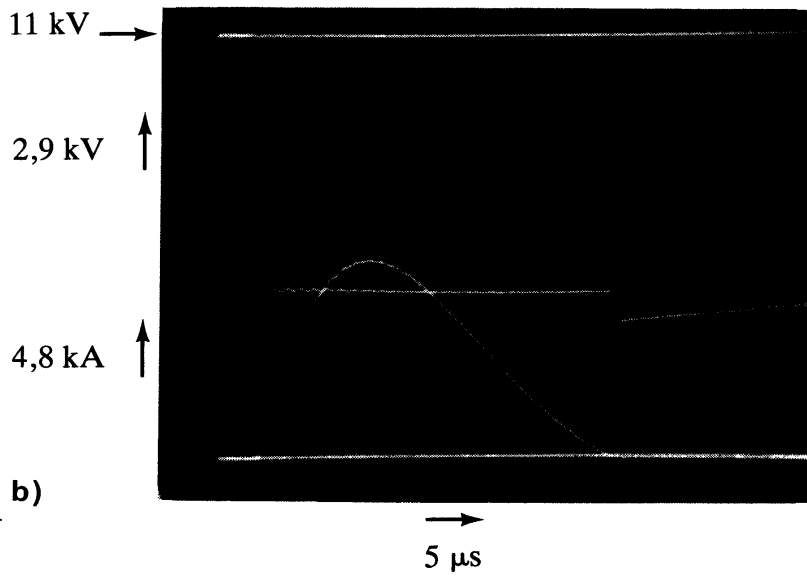

Fig. 3. - Courant d'arc (b) et tension test d'isolement (a) Electrodes en $\mathrm{Cu}$, distance : $3 \mathrm{~mm}$.

[Arc current (b) and recovery voltage test (a) - Cu electrodes, gap $=3 \mathrm{~mm}$.]

Les mesures de courant sont assurées par des sondes de courant à débit interne [12]. Les mesures de tension sont réalisées à partir d'un pont diviseur capacitif et d'un système de transmission opto-électronique [13].

\section{Paramètres considérés.}

Nous avons utilisé des électrodes en molybdène de $56 \mathrm{~mm}$ de diamètre et des électrodes en cuivre OFHC de $66 \mathrm{~mm}$ de diamètre. Nous avons fait varier la distance interélectrodes et nous avons recherché le courant crête critique au-delà duquel un réisolement total n'était plus obtenu.

Nous avons également testé les deux combinaisons d'une association mixte : anode en cuivre et cathode en molybdène et inversement.

\section{Résultats.}

Il apparaît que pour les deux types d'électrodes, le courant critique au-delà duquel nous n'avons plus de réisolement est une fonction décroissante de la distance inter-électrodes (Fig. 6). Dans le cas des électrodes en molybdène, ce claquage apparaît à l'extinction du courant au moment où la tension devient rapidement négative (Fig. 4a). Pour les électrodes en cuivre, ce claquage apparaît de prime abord lors du changement de signe de la tension post-arc de réisolement (Fig. 4b). Nous avons représenté sur la figure 5 les diagrammes synthétiques présentant une coupure réussie (Fig. 5a), et non réussie (Fig. 5b, 5c) pour ces deux types d'électrodes.
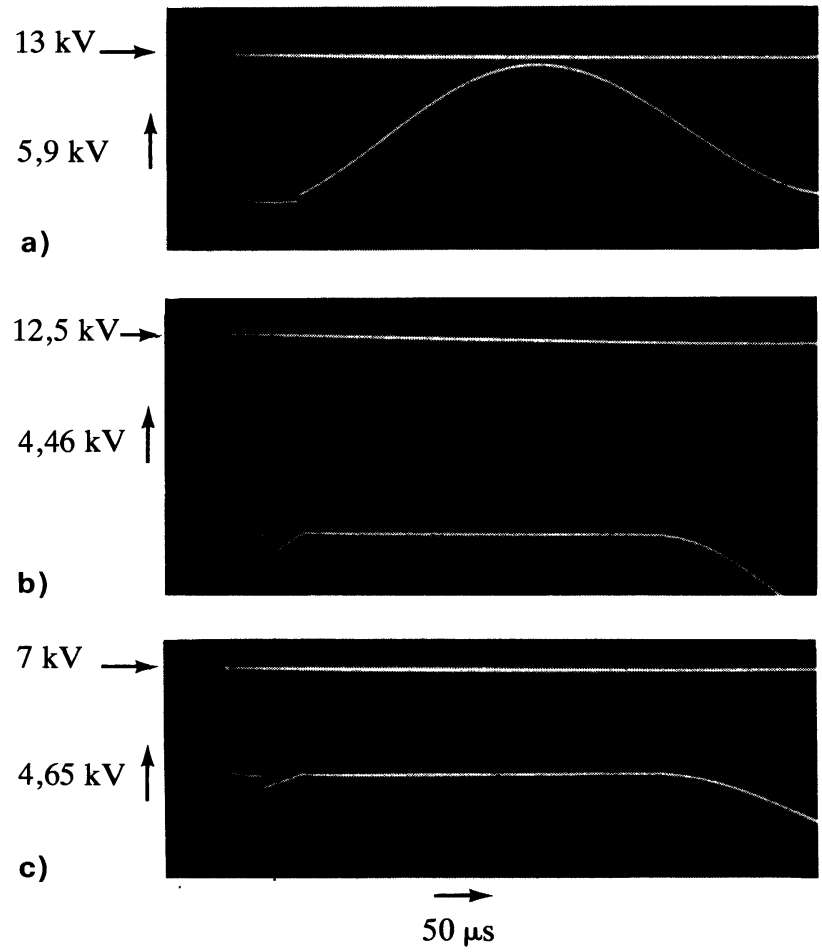

Fig. 4. - Tension test d'isolement : a) Mo $(3 \mathrm{~mm})$; b) $\mathrm{Cu}(2 \mathrm{~mm})$; c) Cathode $\mathrm{Cu}$ et anode $\mathrm{Mo}(2 \mathrm{~mm})$.

[Recovery voltage test : a) Mo $(3 \mathrm{~mm})$; b) Cu (2 mm); c) $\mathrm{Cu}$ cathode and Mo anode $(2 \mathrm{~mm})$.]

Pour une distance inter-électrodes de $2 \mathrm{~mm}$ nous avons obtenu un courant critique de $16 \mathrm{kA}$ (la tension remonte alors de $-1,3 \mathrm{kV}$ à $+12 \mathrm{kV}$ ) dans le cas du molybdène, et de $21 \mathrm{kA}$ (la tension remonte de $-1,7 \mathrm{kV}$ à $+16 \mathrm{kV}$ ) pour le cuivre. Le pic négatif de tension subsiste dans ce dernier cas, jusqu'au passage d'un courant de $27 \mathrm{kA}$ (le pic négatif de tension est alors de $-2,3 \mathrm{kV}$ ).

Dans les deux cas, la cathode est au démontage dotée d'aigrettes globalement radiales, et largement étalées autour du centre (quelques dizaines de millimètres). L'anode se trouve dotée en son centre d'une tache circulaire bleutée de quelques millimètres - sans érosion apparente. L'importance de ces taches est accrue lorsque la distance inter-électrodes diminue. Elles sont ici plus importantes pour les électrodes en cuivre. 

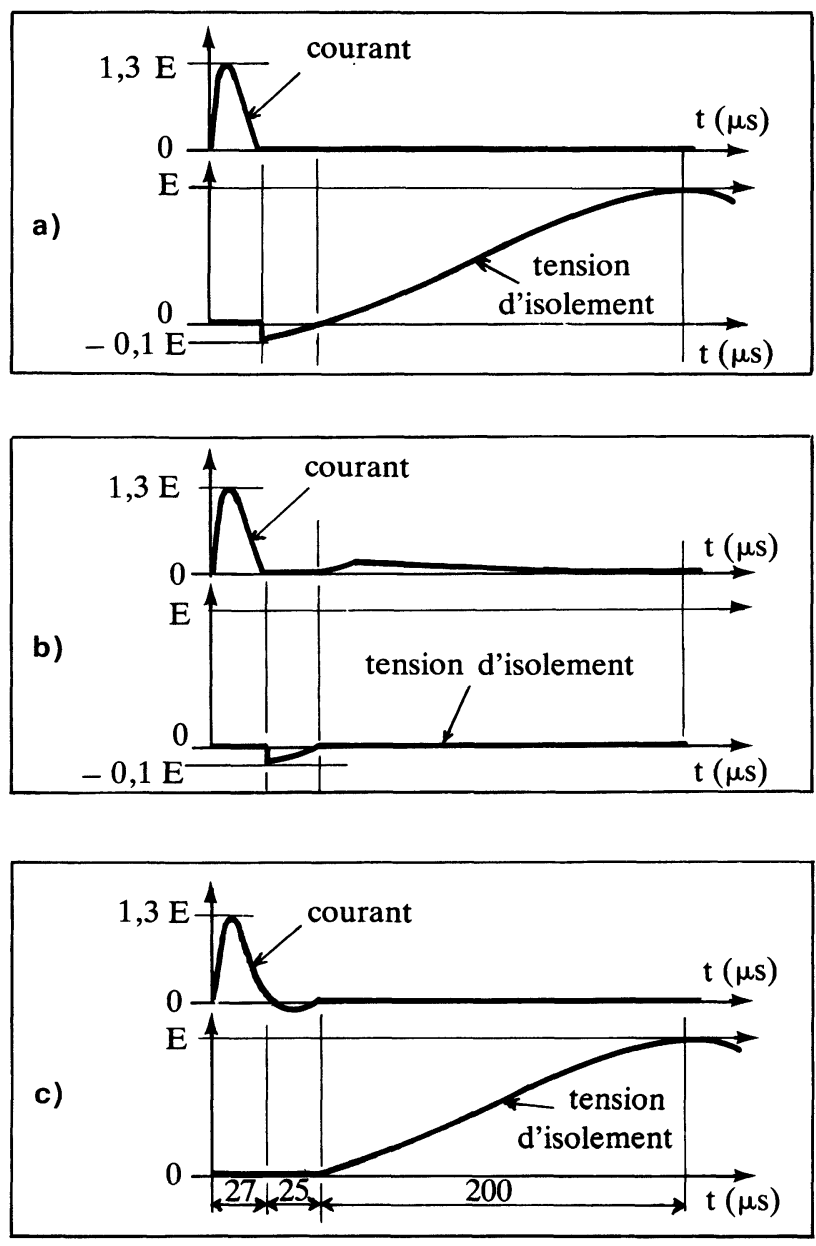

Fig. 5. - Diagrammes synthétiques des coupures: a) Coupure réussie : cas général $(\mathrm{Mo}, \mathrm{Cu})$. b) Coupure non réussie : cas des électrodes en cuivre. c) Coupure non réussie : cas des électrodes en molybdène.

[Interruption synthetic diagrams : a) Successful interruption : general case $(\mathrm{Mo}, \mathrm{Cu}) ; \mathrm{b})$ non successful interruption : copper electrodes case ; c) non successful interruption : molybdenum electrodes case.]

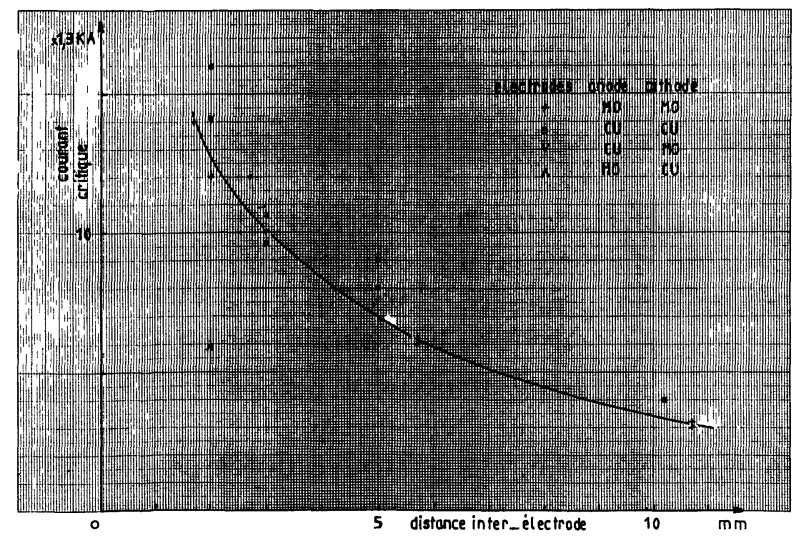

Fig. 6. - Influence de la distance inter-électrodes sur le courant critique.

[Electrode gap influence on the critical current.]
Un trou circulaire de $4 \mathrm{~mm}$ a été réalisé au centre de l'anode des électrodes en cuivre. Dans ce cas les performances ont chuté d'un facteur 4 (distance inter-électrodes $2,7 \mathrm{~mm}$, courant critique $4,5 \mathrm{kA}-$ tension de $-0,5 \mathrm{kV}$ à $+3,5 \mathrm{kV}$ ).

Une autre anode, toujours en cuivre, réduite à un diamètre de $20 \mathrm{~mm}$ (cathode $66 \mathrm{~mm}$ ) permet d'atteindre pour une même distance inter-électrodes $(2,7 \mathrm{~mm})$ un courant critique de $9 \mathrm{kA}$ (la tension post-arc varie de $-770 \mathrm{~V}$ à $+7 \mathrm{kV}$ ). Il ressort par comparaison qu'un rapport de réduction de 3,3 sur le diamètre de l'anode n'apporte qu'une réduction de 1,7 sur le courant critique d'arc.

Nous retrouvons également ces mêmes formes de tache quand nous associons deux électrodes de natures différentes, une en cuivre et l'autre en molybdène.

- Lorsque nous avons l'anode en cuivre et la cathode en molybdène, nous retrouvons des performances équivalentes aux électrodes en molybdène. La rupture d'isolement quand elle intervient est alors totale mais non instantanée.

- Lorsque nous avons l'anode en molybdène, et la cathode en cuivre, les performances chutent fortement. On retrouve cependant le même type de claquage que celui rencontré avec le couple d'électrodes en cuivre (Fig. 4 b, c).

\section{Discussion.}

Il ressort des résultats obtenus que le courant critique au-delà duquel nous n'obtenons plus un bon réisolement est une fonction décroissante de la distance inter-électrodes. Si nous extrapolons ici le rôle des taches anodiques sur les pertes des qualités de réisolement du vide, nous retrouvons la dépendance du courant critique au rapport du diamètre de l'électrode $\Phi$ à la distance inter-électrodes, $l$, explicité ailleurs [1]. Nous avons alors dans notre contexte une relation du type :

$$
\text { (A) } I_{\mathrm{c}}=a\left|\frac{\Phi}{l}\right|^{0,66} \text {. }
$$

Il semble cependant que la tache colorée de l'anode soit une petite surface de fixation de l'arc générée dès l'amorçage et maintenu ensuite par le champ électromagnétique local. Nous notons l'absence de corrosion apparente au niveau de cette tache.

Nous constatons de plus que le même type de rupture d'isolement intervient avec la paire d'électrodes en cuivre et le couple cathode en cuivre et anode en molybdène. Ce claquage a lieu au changement de polarité de la tension test d'isolement. Il est possible que la cathode en cuivre soit à l'origine de la présence de nombreux ions lourds dans l'espace inter-électrodes $[14,15]$. Lors du changement de signe de la tension test, l'inversion dans le mouve- 
ment des populations ioniques serait alors source de claquage. Dans le cas où les électrodes sont exclusivement en molybdène, la population ionique serait moins dense, mais les qualités réfractaires des électrodes deviennent un obstacle majeur à un bon réisolement. Une anode en cuivre améliore sensiblement les performances.

Si maintenant nous nous intéressons au rapport $\alpha$ du courant critique d'arc à la surface des électrodes utilisées (hors arrondi d'extrémité), nous trouvons pour une distance de séparation de $2 \mathrm{~mm}$, une valeur de $9,4 \mathrm{~A} / \mathrm{mm}^{2}$ pour le molybdène et de $8,4 \mathrm{~A} / \mathrm{mm}^{2}$ pour le cuivre. L'activité au niveau des surfaces n'est pas homogène, et il n'est guère possible de préciser la densité de courant réelle admissible au niveau des électrodes. Si nous tenons compte de la relation (A), à diamètre et espacement équivalents, le molybdène serait susceptible d'avoir une valeur de $\alpha$ de $7,3 \mathrm{~A} / \mathrm{mm}^{2}$, alors que le cuivre a une valeur $\alpha$ de $8,4 \mathrm{~A} / \mathrm{mm}^{2}$. Les résultats sont similaires, mais les qualités d'isolement à l'extinction de l'arc lors de l'utilisation d'électrodes en cuivre, laissent à penser qu'une amélioration est possible en faisant intervenir, par exemple, des champs magnétiques axiaux et/ou transverses lors du développement de l'arc [16].

\section{Bibliographie}

[1] Kimblin, C. W., A review of arcing phenomena in vacuum in the transition to atmospheric pressure arcs. IEEE Trans. PS-10 (1982) 322-330.

[2] Miller, H.C., Vacuum arc anode phenomena IEEE Trans. PS-5, 181-196.

[3] Frind, G., Caroll, J. J., Goody, C. P., Tuotty, E. J., Recovery times of vacuum interrupter which have stationnary anodes spots. IEEE Trans. PAS 101 (1982) 775-782.

[4] Rich, J. A., Prescott, L. E., Cobine, J. D., Anode phenomena in metal vapour arcs at high currents. J.A.P. 42 (1971) 587-601.

[5] Kamakshaiah, S., RaU, R. S. N., Anode phenomena in triggered vacuum gaps. IEEE Trans. PS-5 (1977) 1-6.

[6] FARAll, G. A., Recovery of dielectric strength after current interruption in vacuum. IEEE Trans. PS-6 (1978) 360-369.

[7] Zalucki, Z., Seidel, S., Kutzner, J., Contribution à l'étude de la rigidité diélectrique après l'extinction de l'arc dans le vide. III $^{\mathrm{e}}$ Symposium international. Décharges et isolement électrique dans le vide. Paris, sept. 1968.

[8] Harvey, R. J., Lutz, M. A., Gallagher, H. E., Current interruption at powers up to $1 \mathrm{GW}$ with grossed field tubes. IEEE Trans. PS-6 (1978) 248-255.
[9] Kimblin, C. W., Voshall, R. E., Interruption ability of vacuum interrupters subjected to axial magnetic fields. Proc. IEE 119 (1972) 1754-1758.

[10] Rioux, C., Théorie simplifiée des procédés de transfert inductif à haut rendement. Revue Phys. Appl. 10 (1975) 75-79.

[11] Legentil, M., Rioux, C., Caractéristiques des transferts inductifs à haut rendement par procédé dissipatif. Revue Phys. Appl. 11 (1976) 337-342.

[12] Damidau, F., Rioux, C., Etude générale des sondes de mesure inductive de courant. Description des principaux types de sondes. Revue Phys. Appl. 7 (1972) 303-311.

[13] Bleys, C. A., A floatting input optically isolated high voltage. Measurement probe. Rev. Sci. Instrum. 47 (1976) 621-623.

[14] DaAlder, J. E., Components of cathode erosion in vacuum arcs. J. Phys. D 9 (1976) 2379-2394.

[15] Yanabu, S., Homma, M., KaneKo, E., TamaGAWA, T., Post arc current of vacuum interrupters. IEEE Trans. PAS-104 (1985) 166-172.

[16] Yanabu, S., Souma, J., Tamagawa, T., Tsutimi, T., Vacuum arc under an axial magnetic field and its interrupting ability. Proc. IEEE 126 (1979) 313-320. 